\section{Rab3A is essential for mossy fibre long-term potentiation in the hippocampus}

\author{
Pablo E. Castillo ${ }^{\dagger}$, Roger Janz $\ddagger$, Thomas C. Südhof $\ddagger$, \\ Thanos Tzounopoulos $\$$, Robert C. Malenka $\$ \|$ \\ \& Roger A. Nicoll $* \|$
}

Departments of ${ }^{\star}$ Cellular and Molecular Pharmacology, $\$$ Psychiatry and \|Physiology, University of California, San Francisco, California 94143, USA $\$$ Department of Molecular Genetics and Howard Hughes Medical Institute, The University of Texas Southwestern Medical Center, Dallas, Texas 75235, USA $\dagger$ Present address: Departamento de Fisiologia, Facultad de Medicina, Gral Flores 2125, Montevideo, Uruguay.

Repetitive activation of excitatory synapses in the central nervous system results in a long-lasting increase in synaptic transmission called long-term potentiation (LTP). It is generally believed that this synaptic plasticity may underlie certain forms of learning and memory. LTP at most synapses involves the activation of the NMDA ( $N$-methyl-D-aspartate) subtype of glutamate receptor, but LTP at hippocampal mossy fibre synapses is independent of NMDA receptors and has a component that is induced and expressed presynaptically ${ }^{1}$. It appears to be triggered by a rise in presynaptic $\mathrm{Ca}^{2+}$ (refs 2,3 ), and requires the activation of protein kinase $\mathrm{A}^{4-6}$, which leads to an increased release of glutamate ${ }^{3,7-10}$. A great deal is known about the biochemical steps involved in the vesicular release of transmitter ${ }^{1-13}$, but none of these steps has been directly implicated in long-term synaptic plasticity. Here we show that, although a variety of short-term plasticities are normal, LTP at mossy fibre synapses is abolished in mice lacking the synaptic vesicle protein Rab3A.

In searching for membrane fusion proteins that might be involved in mossy fibre LTP, a starting point is to look at those proteins that are substrates for protein kinase A (PKA). The most thoroughly studied synaptic proteins that are phosphorylated by
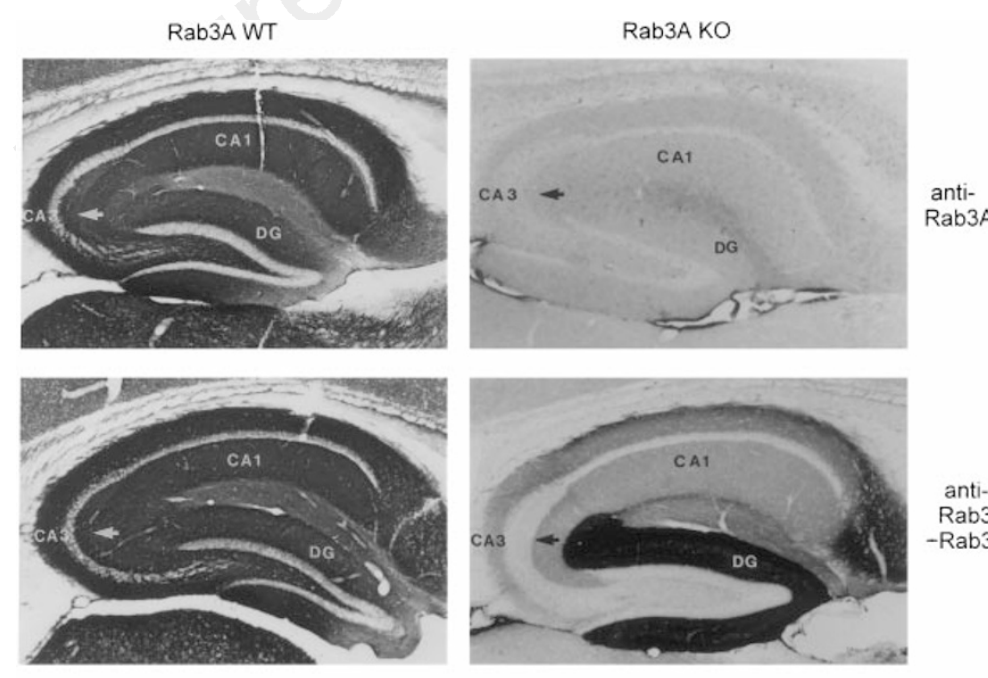

Rab3A
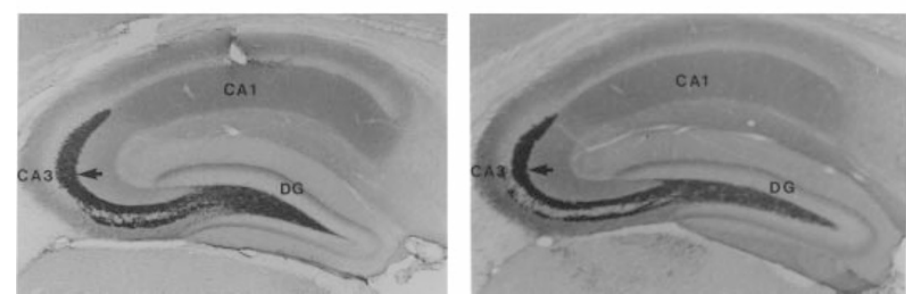

PKA are the synapsins ${ }^{14}$. However, previous studies on mutant mice in which both synapsin I and II were deleted showed normal LTP ${ }^{15}$. Another vesicle-associated protein, rabphilin-3, is also a substrate for $\mathrm{PKA}^{16}$. Rabphilin exerts its effects by binding to Rab3 proteins (specifically Rab3A and Rab3C at synapses), which are small, GTPbinding proteins that are thought to attach reversibly to the membrane of the synaptic vesicle ${ }^{13}$. Furthermore, a second putative Rab3-effector protein, called Rim, was recently discovered ${ }^{17}$ that also contains PKA phosphorylation consensus sequences. This suggests that Rab3A and Rab3C may have multiple targets at the synapse that are potentially regulated by PKA. Previous electrophysiological studies in the CA1 region of the hippocampus from mice lacking the Rab3A protein showed that, although most synaptic parameters were normal, there is increased synaptic depression after short trains of repetitive stimuli ${ }^{18}$. Here we consider the physiological properties of mossy fibre synapses that exhibit short- and long-term plasticities that are profoundly different from those previously studied in the brain.

Initially we used highly sensitive immunoperoxidase labelling ${ }^{19}$ to examine the anatomy of the mossy fibre pathway in Rab3A-knockout mice and in littermates. Monoclonal antibodies specific for Rab3A (C142.2) or cross reactive with all Rab3 isoforms in brain (Rab3A, 3B and 3C; C142.1) and polyclonal antibodies to Rab3C were used to evaluate Rab3 proteins in the hippocampus (Fig. 1 and data not shown). Immunoperoxidase staining failed to reveal the presence of Rab3C or Rab3B in the stratum lucidum, the termination zone of the mossy fibres. In contrast, high levels of Rab3C were observed in the rest of the hippocampus, especially in the dentate gyrus, which is the termination zone of fibres from the entorhinal cortex. Thus Rab3A is the only detectable Rab3 isoform in the mossy fibre terminals, making this synapse an excellent system in which to study the effect of the Rab3A knockout in the absence of possibly redundant Rab3 isoforms. No induction of other Rab3 proteins or changes in the structure of the mossy fibre layer was observed in the Rab3A-knockout mice. When synaptoporin/synaptophysin II was used as a marker for mossy fibre terminals ${ }^{20}$, no changes in the intensity of staining or the structure of stratum lucidum was apparent (Fig. 1). Together with the previously characterized lack of ultrastructural changes in Rab3A-knockout mice ${ }^{18}$, these data 
suggest that the mossy fibre pathway in these mice is structurally normal but lacks other Rab3 proteins, making it uniquely suited for the study of the effects of the Rab3A knockout on synaptic function and plasticity.

Although no anatomical abnormalities could be detected, it is possible that the Rab3A deletion prevents the release of transmitter from mossy fibre synapses. Testing the functional integrity of the mossy fibre pathway is not trivial as, in the absence of functional mossy fibres, activation of neighbouring associational/commissural fibres would still be expected to elicit a response. Thus a method was required to distinguish mossy fibre synaptic responses from nonmossy fibre synaptic responses. We have found, in agreement with previous observations ${ }^{21,22}$, that the mossy fibre synapses in the mouse possess inhibitory presynaptic metabotropic glutamate receptors (mGluRs) of the group 2 subtype, whereas associational/commissural fibres do not have these receptors. Thus application of the group 2-selective agonist L-CCG1 $(10 \mu \mathrm{M})$ had no effect on the responses to associational/commissural stimulation but completely blocked the response evoked by a stimulating electrode placed in the dentate granule cell layer (Fig. 2a). The magnitude of the inhibition induced by L-CCG1 was the same in wild-type and Rab3A-deficient mice (Fig. 2b), indicating that the mossy fibres are present and functional in the knockout animal.

We next looked for possible effects on two forms of short-term synaptic plasticity of knocking out Rab3A. When a synapse is activated twice at short intervals, the size of the response to the second stimulus is increased, as there is an increase in transmitter release $^{23}$. This is referred to as paired-pulse facilitation (PPF). In the mossy fibres, tested at a 40-ms interstimulus interval with fieldpotential recording, PPF exhibited no significant change in the knockout (wild type, $2.7 \pm 0.3, n=15$; knockout, $3.0 \pm 0.4$, $n=14)$. Because PPF in the CA1 region is enhanced in the Rab3A-deficient mouse ${ }^{24}$, especially at short intervals, we repeated our studies on mossy fibre synapses using the whole-cell voltageclamp technique. No difference in PPF was found either at 20 or
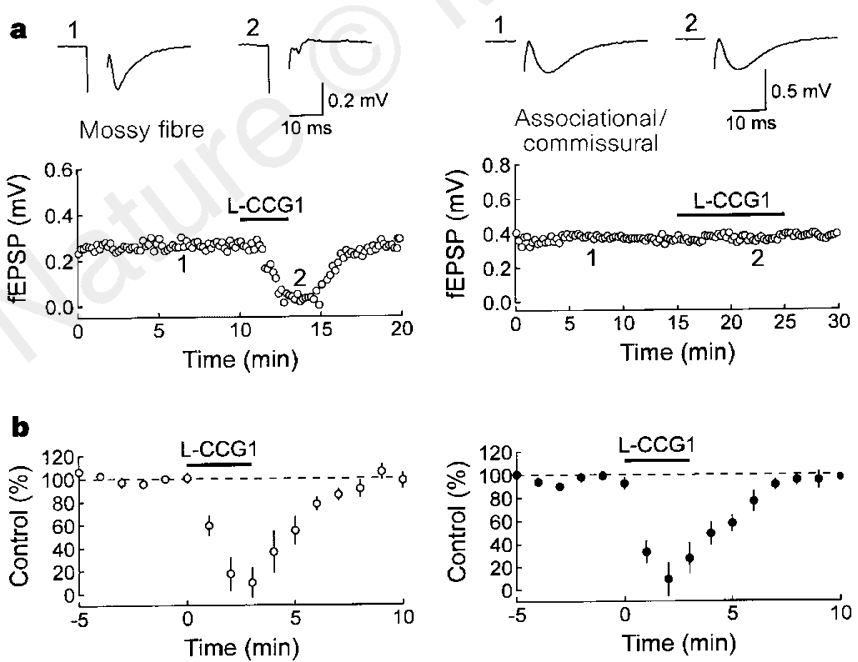

Figure 2 Mossy fibre synaptic inputs are normal in Rab3A-deficient mice. a, LCCG1 $(10 \mu \mathrm{M})$ bath application for $3 \mathrm{~min}$ abolishes mossy fibre synaptic responses (left); longer application (10 $\mathrm{min}$ ) has no effect on associationalcommissural responses (right). b, Inhibitory effects of L-CCG1 were identical in wild-type (left, open circles; 4, animals, 6 slices) and Rab3A-mutant mice (right, filled circles; 4 animals, 7 slices), indicating that functional mossy fibre inputs are intact in these mice. fEPSP, field excitatory postsynaptic potential.
$40 \mathrm{~ms}$ (Fig. 3a). As well as exhibiting robust PPF, mossy fibre responses are also extremely sensitive to the frequency of stimulation, such that modest increases in stimulation frequency cause a large increase in transmitter release ${ }^{25,26}$. This phenomenon, referred to as frequency facilitation ${ }^{23}$, was unaffected in the knockout mouse (Fig. 3b). Thus two different forms of short-term synaptic plasticity were unaffected by the deletion of Rab3A. Both of these forms of plasticity are very sensitive to the probability of transmitter release, consistent with the idea that the probability of release is not markedly altered in the knockout mice. However, our experiments do not address the possibility that the amount of transmitter release, once release occurs, is increased or decreased in the knockout mice.

We next examined the increase in synaptic transmission evoked by raising the levels of cyclic AMP by applying forskolin. This enhancement of mossy fibre transmission is primarily the result of an increased release of neurotransmitter ${ }^{4,5}$, and was also unaffected in the knockout (Fig. 3c). To confirm that the forskolin-induced enhancement was due to an elevation in cAMP, we carried out two further experiments. First, we examined the effects of Sp-8-Br-CPTcAMPS, a membrane-permeant analogue of cAMP, on mossy fibre responses. The increase in transmission caused by $\mathrm{Sp}-8$-Br-CPTcAMPS was similar in the wild-type ( $179 \pm 15 \%$ of control, $n=3)$ and the knockout mice (187 $\pm 12 \%$ of control, $n=4)$. Second, we found that an analogue of forskolin, 1,9-dideoxyforskolin, which does not activate adenylyl cyclase but shares some of the other effects of forskolin ${ }^{27}$, had no effect on mossy fibre responses $(n=3)$.

Finally, we tested for possible effects of the knockout on mossy fibre LTP. Tetanic stimulation (given in the presence of $100 \mu \mathrm{M} D$ AP5) elicited robust mossy fibre LTP in wild-type mice. In marked contrast, LTP was essentially absent in the Rab3A-deficient mice (Fig. 4). The potentiation immediately after the tetanus, however, did not seem to be altered in the knockout. In addition, the size of the synaptic response at the end of the tetanus was similar in the wild-type ( $46 \pm 20 \%$ of pre-tetanus response, $n=6$ ) and knockout mice ( $36 \pm 18 \%, n=6$ ) (not shown). These results suggest that, on
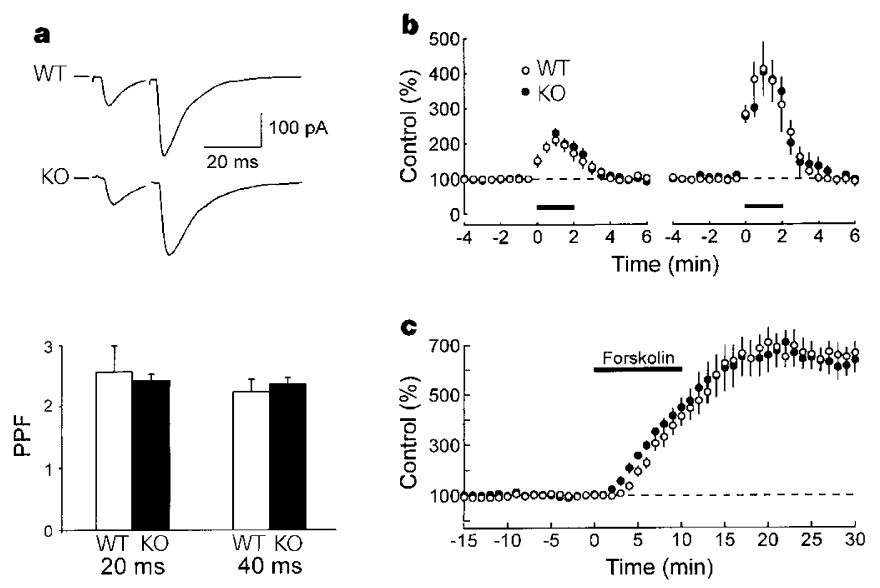

Figure 3 Synaptic transmission in Rab3A-deficient mice. a, Paired-pulse facilitation (PPF) measured at intervals of 20 and $40 \mathrm{~ms}$ was compared in normal and mutant mice using whole-cell voltage-clamp techniques. Top, typical responses from wild-type (WT) and Rab3A-knockout (KO) mice. Bottom, magnitude of PPF (mean \pm s.e.m.) at 20 and $40 \mathrm{~ms}$ is summarized for wild-type (white bars; 3 animals, 8 cells) and knockout mice (black bars; 3 animals, 9 cells). b. Frequency facilitation was explored with field-potential recording in wild-type (open circles; 5 animals, 8 slices) and mutant mice (filled circles; 6 animals, 10 slices) by changing the frequency of stimulation for $2 \mathrm{~min}$ (horizontal bars) from 0.1 to $0.2 \mathrm{~Hz}$ (left) or 0.1 to $0.33 \mathrm{~Hz}$ (right). There are no significant differences $(P>0.5)$ in PPF or frequency facilitation between wild-type and mutant mice. c, Bath application of forskolin ( $10 \mu \mathrm{M}$ for $3 \mathrm{~min}$ ) induced the same magnitude of potentiation in wild-type (white circles; 5 animals, 8 slices) and Rab3A-mutant mice (black circles; 5 animals, 7 slices). 

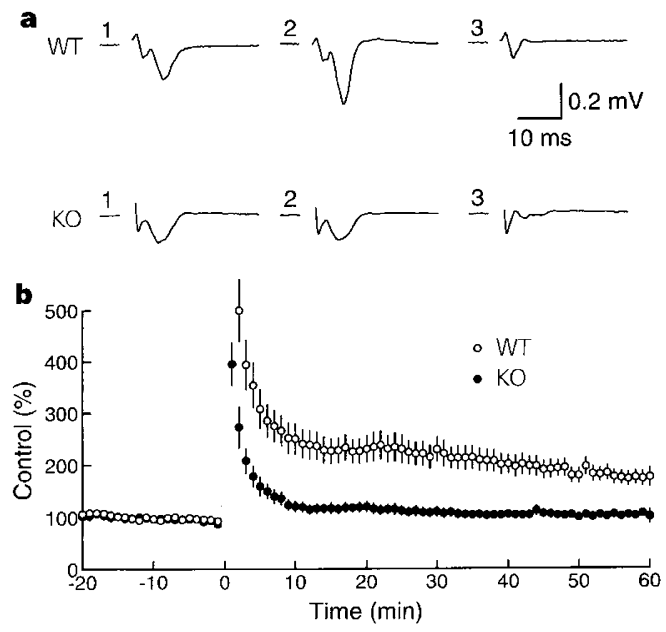

Figure 4 Mossy fibre LTP is blocked in Rab3A-deficient mice. a, Sample traces before (1), 55-60 min after tetanization (2) and after $10 \mu \mathrm{M}$ L-CCG1 bath application (3) in wild-type (WT, top) and knockout (KO, bottom) mice. b, LTP summary graphs (mean \pm s.e.m.) in wild-type (white circles; 9 animals, 13 slices) and mutant mice (black circles; 10 animals, 13 slices). LTP was induced by one train lasting $5 \mathrm{~s}$ at $25 \mathrm{~Hz}$ in the presence of $100 \mu \mathrm{M}$ D-AP5.

average, the synapses in the two groups were activated to a similar extent by the tetanus.

Thus, in Rab3A-knockout mice, synaptic plasticity at the mossy fibre synapses exhibits a selective phenotype: LTP cannot be induced. All of the other synaptic parameters investigated were normal, suggesting that the phenotype is discrete. These data show that Rab3A is essential for mossy fibre LTP. To our knowledge, this observation provides the first example of a synaptic protein that is involved in vesicular transport and is essential for long-term synaptic plasticity, and supports the idea that mossy fibre LTP is a presynaptic modification operating at the secretory apparatus.

How might such a selective defect in mossy fibre LTP be explained? One model for mossy fibre $\mathrm{LTP}^{1,3}$ proposes that, during tetanic stimulation, $\mathrm{Ca}^{2+}$ accumulates in the presynaptic terminal and activates a $\mathrm{Ca}^{2+} /$ calmodulin-sensitive adenylyl cyclase. The resulting rise in cAMP activates PKA, which in turn phosphorylates substrate protein(s) which, in ways yet to be defined, increase the evoked release of transmitter. cAMP has also been shown to increase release at several other synapses ${ }^{28,29}$. One possible explanation for the absence of mossy fibre LTP is that, in the Rab3Adeficient mouse, adenylyl cyclase is not activated because the tetanus-induced rise in presynaptic $\mathrm{Ca}^{2+}$ has been impaired. This seems unlikely, however, because three different $\mathrm{Ca}^{2+}$-dependent forms of short-term plasticity at mossy fibre synapses (PPF, frequency facilitation and post-tetanic potentiation) were unaffected by the knockout. Another possibility is that Rab3A is involved in keeping adenylyl cyclase near the $\mathrm{Ca}^{2+}$ channels. In the absence of $\mathrm{Rab} 3 \mathrm{~A}, \mathrm{Ca}^{2+}$ would no longer be able to activate the cyclase, but forskolin would still be effective in enhancing release because the cyclase is still present in the terminal. Although this hypothesis cannot formally be excluded, there is no evidence suggesting an interaction between Rab3A (or its associated proteins) and adenylyl cyclase.

A more likely possibility is that the Rab3A deletion prevents PKAmediated modulation of some step in the release process itself. In this regard, the observation that Rab3A may regulate the number of vesicles that are released as a function of $\mathrm{Ca}^{2+}$ is important ${ }^{24}$. This observation suggests a model by which LTP could occur, namely by changing the gain on the $\mathrm{Ca}^{2+}$ signal mediated by Rab3A. This hypothesis agrees well with the observation that both putative effectors for Rab3A, rabphillin and Rim, have PKA consensus phosphorylation sites ${ }^{16,17}$. According to this model, LTP would normally partially inactivate Rab3A, possibly by a phosphorylationdependent action on its effectors, and no such inactivation would be possible in the absence of Rab3A. At this point, it is unclear whether Rab3A acts through rabphillin and/or Rim, or an as-yet unidentified effector. It is possible that rabphillin alone mediates mossy fibre LTP, and that the mislocation ${ }^{30}$ and subsequent loss of rabphillin in the Rab3A-deficient mouse is the underlying cause for the mossy fibre LTP phenotype. Although the finding that PPF is unaltered at mossy fibre synapses but increased at CA1 excitatory synapses ${ }^{24}$ might indicate a different role for Rab3A at these two types of synapse, the greater magnitude of PPF at mossy fibre synapses may mask the effects seen at CA1 synapses.

It is surprising that short-term synaptic plasticity and forskolininduced enhancement are intact in the Rab3A-knockout mouse. This is unexpected because induction of mossy fibre LTP partly occludes $\mathrm{PPF}^{5,7}$, frequency facilitation ${ }^{26}$ and forskolin enhancement ${ }^{4}$. Although it cannot be ruled out that the involvement of Rab3A in mossy fibre LTP is independent of cAMP, the most plausible explanation for this apparent paradox is that induction of mossy fibre LTP normally also occludes short-term synaptic plasticity and forskolin enhancement by a mechanism that is parallel to, but not identical to, LTP induction. This explanation is supported by the observation that forskolin enhancement was also normal in mice that exhibited a loss of mossy fibre LTP as a result of mutations in the $\mathrm{C} \beta_{1}$ and $\mathrm{RI} \beta$ isoforms of $\mathrm{PKA}^{6}$.

In summary, we have found that knocking out Rab3A causes a remarkably selective functional defect at mossy fibre synapses: it abolishes mossy fibre LTP. This finding not only supports the presynaptic locus of mossy fibre LTP, but also provides the first link between a protein of the secretory machinery at the synapse and a form of long-term synaptic plasticity. Finally, our results are consistent with a model in which the increase in transmitter release during mossy fibre LTP occurs at a late step in the secretory process.

\section{Methods}

Generation of the Rab3A-mutant mouse lines was described previously ${ }^{18}$. All data were acquired from homozygous mutant and wild-type littermates from heterozygote matings; heterozygotes were excluded. Hippocampal slices were prepared from mice 6 to 12 weeks old. The composition of the external recording solution was (in $\mathrm{mM}$ ): $119 \mathrm{NaCl}, 2.5 \mathrm{KCl}, 1.2 \mathrm{MgSO}_{4}, 2.5 \mathrm{CaCl}_{2}$, $1.0 \mathrm{NaHPO}_{4}, 26.2 \mathrm{NaHCO}_{3}$ and 10 glucose. Extracellular field recording and whole-cell patch-clamp recording ${ }^{2}$ were performed at room temperature. For the whole-cell experiments, picrotoxin $(100 \mu \mathrm{M})$ was present to block inhibition, and $0.3 \mu \mathrm{M}$ CNQX and $50 \mu \mathrm{M}$ D-AP5 were added to prevent epileptiform discharge. Mice were shipped to San Francisco unidentified, and data were acquired and analysed by the experimenter in a blind fashion. After the experiment, the genotype was confirmed by PCR and western blot analysis using tail DNA and brain protein. Drugs used were D-2-amino-5-phosphonovaleric acid (D-AP5), (2S,3S,4S)-CCG/(2S,1'S,2'S)-2-(carboxycyclopropyl)glycine (L-CCG1), 6-cyano-7-nitroquinoxaline-2,3-dione (CNQX) (TocrisCookson), picrotoxin (Sigma) and forskolin (CalBiochem). The data are expressed as mean \pm s.e.m. as a percentage of the baseline. Student's $t$-test was used to determine whether there was a significant difference in the means between the results from wild-type and mutant mice. For the anatomy presented in Fig. 1, sections of wild-type and Rab3A-deficient mice littermates (6 weeks old) were immunostained with the following antibodies: C142.2 (monoclonal antibody specific for Rab3A), C142.1 (monoclonal antibody specific for Rab3A/B/C), and a polyclonal antibody specific for synaptophysin II. The dilutions used were $1 / 2,000$ to $1 / 5,000$ of ascites or serum.

Received 28 February; accepted 13 June 1997.

1. Nicoll, R. A. \& Malenka, R. C. Contrasting properties of two forms of long-term potentiation in the hippocampus. Nature 377, 115-118 (1995).

2. Castillo, P. E., Weisskopf, M. G. \& Nicoll, R. A. The role of $\mathrm{Ca}^{2+}$ channels in hippocampal mossy fiber synaptic transmission and long-term potentiation. Neuron 12, 261-269 (1994).

3. Tong, G., Malenka, R. C. \& Nicoll, R. A. Long-term potentiation in cultures of single hippocampal granule cells: A presynaptic form of plasticity. Neuron 16, 1147-1157 (1996). 
4. Weisskopf, M. G., Castillo, P. E., Zalutsky, R. A. \& Nicoll, R. A. Mediation of hippocampal mossy fiber long-term potentiation by cyclic AMP. Science 265, 1878-1882 (1994).

5. Huang, Y-Y., Li, X.-C. \& Kandel, E. cAMP contributes to mossy fiber LTP by initiating both a covalently mediated early phase and macromolecular synthesis-dependent late phase. Cell 79, 69-79 (1994).

6. Huang, Y. Y. et al. A genetic test of the effects of mutations in PKA on mossy fiber LTP and its relation to spatial and contextual learning. Cell 83, 1211-1222 (1995).

Zalutsky, R. A. \& Nicoll, R. A. Comparison of two forms of long-term potentiation in single hippocampal neurons. Science 248, 1619-1624 (1990).

8. Weisskopf, M. G. \& Nicoll, R. A. Presynaptic changes during mossy fiber LTP revealed by NMDA receptor-mediated synaptic responses. Nature 376, 256-259 (1995)..

9. López-García, J. C., Arancio, O., Kandel, E. R. \& Baranes, D. A presynaptic locus for long-term potentiation of elementary synaptic transmission at mossy fiber synapses in culture. Proc. Natl Acad. Sci. USA 93, 4712-4717 (1996).

10. Xiang, Z., Greenwood, A. C., Kairiss, E. W. \& Brown, T. H. Quantal mechanism of long-term potentiation in hippocampal mossy-fiber synapses. J. Neurophysiol. 71, 2552-2556 (1994).

11. Ferro-Novick, S. \& Jahn, R. Vesicle fusion from yeast to man. Nature 370, 191-193 (1994).

12. Bennett, M. K. \& Scheller, R. H. A molecular description of synaptic vesicle membrane trafficking. Annu. Rev. Biochem. 63, 63-100 (1994).

13. Südhof, T. C. The synaptic vesicle cycle: a cascade of protein-protein interactions. Nature 375, $645-$ 653 (1995).

14. DeCamilli, P., Benfenati, F., Valtorta, F. \& Greengard, P. The synapsins. Annu. Rev. Cell Biol. 6, $433-$ 460 (1990)

15. Spillane, D. M., Rosahl, T. W., Südhof, T. C. \& Malenka, R. C. Long-term potentiation in mice lacking synapsins. Neuropharmacology 34, 1573-1579 (1995).

16. Fykse, E. M., Li, C. \& Südhof, T. C. Phosphorylation of rabphilin-3A by $\mathrm{Ca}^{2+} / \mathrm{calmodulin-}$ and cAMPdependent protein kinases in vitro. J. Neurosci. 15, 2385-2395 (1995).

17. Wang, Y., Okamoto, M., Schmitz, F., Hofmann, K. \& Südhof, T. C. Rim is a putative Rab3 effector in regulating synaptic-vesicle fusion. Nature 387, 000-000 (1997)

18. Geppert, M. et al. The role of Rab3A in neurotransmitter release. Nature 369, 493-497 (1994)

19. Rosahl, T. W. et al. Essential functions of synapsins I and II in synaptic vesicle regulation. Nature 375, 488-493 (1995)

20. Fykse, E. M. et al. Relative properties and localizations of synaptic vesicle protein isoforms: the case of the synaptophysins. J. Neurosci. 13, 4997-5007 (1993).

21. Kamiya, H., Shinozaki, H. \& Yamamoto, C. Activation of metabotropic glutamate receptor type $2 / 3$ suppresses transmission at rat hippocampal mossy fibre synapses. J. Physiol. (Lond.) 493, 447-455 (1996)

22. Yokoi, M. et al. Impairment of hippocampal mossy fiber LTD in mice lacking mGluR2. Science $\mathbf{2 7 3}$ 645-647 (1996)

23. Zucker, R. S. Short-term synaptic plasticity. Annu. Rev. Neurosci. 12, 13-31 (1989).

24. Geppert, M., Goda, Y., Stevens, C. F. \& Südhof, T. C. Rab3A regulates a late step in synaptic vesicle fusion. Nature 387, 810-814 (1997).

25. Regehr, W. G., Delaney, K. R. \& Tank, D. W. The role of presynaptic calcium in short-term enhancement at the hippocampal mossy fiber synapse. J. Neurosci. 14, 523-537 (1994).

26. Salin, P. A., Scanziani, M., Malenka, R. C. \& Nicoll, R. A. Distinct short-term plasticity at two excitatory synapses in the hippocampus. Proc. Natl Acad. Sci. USA 93, 13304-13309 (1996).

27. Seamon, K. B. \& Daly, J. W. Forskolin: Its biological and chemical properties. Adv. Cyclic Nucleot. Prot. Phosphoryl. Res. 20, 1-150 (1986)

28. Dixon, D. \& Atwood, L. Adenylate cyclase system is essential for long-term facilitation at the crayfish neuromuscular junction. $J$. Neurosci. 9, 4246-4252 (1989).

29. Schacher, S., Castellucci, V. F. \& Kandel, E. R. cAMP evokes long-term facilitation in Aplysia sensory neurons that requires new protein synthesis. Science 240, 1667-1669 (1988).

30. $\mathrm{Li}$, C. et al. Synaptic targeting of rabphilin-3A, a synaptic vesicle $\mathrm{Ca}^{2+} /$ phospholipid-binding protein depends on rab3A/3C Neuron 13,885-898 (1994).

Acknowledgements. We thank H. Czerwonka for secretarial assistance. R.A.N. is a member of the Keck Center for Integrative Neuroscience and the Silvio Conte Center for Neuroscience Research. R.C.M. is member of the Center for Neurobiology and Psychiatry, and the Center for the Neurobiology of Addiction. R.A.N. and R.C.M. are supported by grants from the NIH. T.C.S. was supported by grants from the HFJP and the W.M. Keck Foundation. R.J. was supported by a postdoctoral fellowship from DFG.

Correspondence and requests for materials should be addressed to R.A.N. (e-mail: nicoll@phy.ucsf.edu).

\section{Rim is a putative Rab3 effector in regulating synaptic-vesicle fusion}

\section{Yun Wang ${ }^{\star}$, Masaya Okamoto*, Frank Schmitz $\dagger$ Kay Hofmann $\ddagger$ \& Thomas C. Südhof*}

${ }^{\star}$ Department of Molecular Genetics and Howard Hughes Medical Institute, The University of Texas, Southwestern Medical Center at Dallas, Texas 75235, USA

$\dagger$ Abteilung IX, Max-Planck-Institut für Experimentelle Medizin,

37075 Göttingen, Germany

$\ddagger$ Swiss Institute for Experimental Cancer Research, 1066 Epalinges, Switzerland

Rab3 is a neuronal GTP-binding protein that regulates fusion of synaptic vesicles and is essential for long-term potentiation of hippocampal mossy fibre synapses ${ }^{1-5}$. More than thirty Rab GTPbinding proteins are known to function in diverse membrane transport pathways, although their mechanisms of action are unclear. We have now identified a putative Rab3-effector protein called Rim. Rim is composed of an amino-terminal zinc-finger motif and carboxy-terminal PDZ and $\mathrm{C}_{2}$ domains. It binds only to GTP (but not to GDP)-complexed Rab3, and interacts with no other Rab protein tested. There is enrichment of Rab3 and Rim in neurons, where they have complementary distributions. Rab3 is found only on synaptic vesicles, whereas Rim is localized to presynaptic active zones in conventional synapses, and to presynaptic ribbons in ribbon synapses. Transfection of PC12 cells with the amino-terminal domains of Rim greatly enhances regulated exocytosis in a Rab3-dependent manner. We propose that Rim serves as a Rab3-dependent regulator of synaptic-vesicle fusion by forming a GTP-dependent complex between synaptic plasma membranes and docked synaptic vesicles.

Rab3A and Rab3C are small GTP-binding proteins of synaptic vesicles that cycle between vesicle-associated, GTP-complexed forms and cytosolic, GDP-complexed forms as a function of exocytosis ${ }^{6-8}$. Deletion of Rab3A in mice enhances neurotransmitter release without changing the docking or priming of synaptic vesicles, suggesting that Rab3 has a role in regulating synapticvesicle fusion ${ }^{4}$. Rab3A is also required for long-term potentiation (LTP) of synaptic transmission in mossy fibre synapses of the hippocampus, indicating that Rab3 physiologically regulates the extent of synaptic-vesicle fusion in synaptic plasticity ${ }^{5}$. Rab3 and other Rab proteins are thought to function by GTP-dependent interactions with effector proteins ${ }^{8-10}$, although the number and nature of these effectors are unknown: only a few candidate effectors have been described for more than thirty Rab proteins, including rabphilin-3 $\mathrm{A}^{11}$. Rabphilin is colocalized with Rab3 on synaptic vesicles, and binds to Rab3 as a function of GTP by means of an $\mathrm{N}$-terminal zinc-finger domain ${ }^{12,13}$. However, rabphilin on its own seems insufficient to account for the actions of Rab3, and so Rab3 could have multiple effectors.

By using an activating form of Rab3C fused to the DNA-binding domain of LexA as a bait, we screened a rat brain cDNA library by yeast two-hybrid selection for proteins that interact with Rab3. Positive prey clones were rescued and retransformed into fresh yeast cells with Rab3 baits or control baits. We then evaluated the specificity of the prey and bait interactions by using two assays for transactivation, namely $\beta$-galactosidase induction and histidine autotrophy ${ }^{14}$. In this way we identified a class of prey clones that interact only with Rab3 in the yeast two-hybrid system, and encode part of a new protein called Rim (for Rab3-interacting molecule). Northern blots revealed that Rim mRNA is only detectable in brain (data not shown), suggesting that Rim is a candidate neuronal effector for the synaptic actions of Rab3.

We obtained only partial Rim cDNA clones in the yeast twohybrid screens, so we isolated full-length cDNA clones from rat brain libraries. Their sequences showed that Rim is a large protein (1,553 amino acids) with several alternatively spliced variants (Fig. 1). We observed variable sequences in multiple independent cDNA clones at three positions. Two of these alternatively spliced sequences occur in three distinct variants, suggesting that Rim can be present in up to 18 forms. Databank searches identified a Rim homologue in Caenohhabditis elegans that is distributed over two cosmid sequences (T10A3 and K03A1). Because rabphilin is also present in $C$. elegans, both putative effectors for Rab3 are evolutionarily conserved. Analysis of the Rim sequence demonstrates that it is composed of multiple domains present in both the rat and C. elegans proteins: an N-terminal zinc-finger domain, a highly charged sequence followed by an alanine/proline-rich region, a PDZ domain, and $\mathrm{C}_{2}$ domains separated by alternatively spliced sequences (Fig. 1).

The Rab3-binding protein rabphilin also has a zinc-finger motif and two $\mathrm{C}_{2}$ domains. However, the zinc-fingers of Rim and rabphilin are only weakly homologous (42\% identity over 55 residues), and their $\mathrm{C}_{2}$ domains belong to different classes ${ }^{15}$. Furthermore, rabphilin lacks the other domains of Rim (the PDZ 